\title{
Diacronie
}

Studi di Storia Contemporanea

$N^{\circ} 36,4 \mid 2018$

Viaggi e turismo nell'Europa del Novecento

\section{Gabriele Donato, La violenza, la rivolta. Cronologia della lotta armata in Italia 1966-1988}

\section{Alessandro Chebat}

\section{Q OpenEdition}

Edizione digitale

URL: https://journals.openedition.org/diacronie/10499

DOI: 10.4000/diacronie.10499

ISSN: 2038-0925

Editore

Association culturelle Diacronie

Notizia bibliografica digitale

Alessandro Chebat, «Gabriele Donato, La violenza, la rivolta. Cronologia della lotta armata in Italia

1966-1988», Diacronie [Online], № 36, 4 | 2018, documento 17, online dal 29 décembre 2018, consultato il 17 février 2022. URL: http://journals.openedition.org/diacronie/10499; DOI: https://doi.org/10.4000/ diacronie. 10499 


\title{
Diacronie
}

Studi di Storia Contemporanea

$36,4 / 2018$

Viaggi e turismo nell'Europa del Novecento

\section{RECENSIONE: Gabriele DONATO, La violenza, la rivolta. Cronologia della lotta armata in Italia 1966-1988, Trieste, IRSML, 2018, 417 pp.}

\author{
A cura di Alessandro CHEBAT
}

Per citare questo articolo:

CHEBAT, Alessandro, «RECENSIONE: Gabriele DONATO, La violenza, la rivolta. Cronologia della lotta armata in Italia 1966-1988, Trieste, IRSML, 2018, 417 pp.», Diacronie. Studi di Storia Contemporanea : Viaggi e turismo nell'Europa del Novecento, 36, 4/2018, 29/12/2018,

URL: < http://www.studistorici.com/2018/12/29/chebat_numero_36/ >

Diacronie Studi di Storia Contemporanea $\rightarrow$ http://www.diacronie.it

Rivista storica online. Uscita trimestrale.

redazione.diacronie@hotmail.it

Comitato di direzione: Naor Ben-Yehoyada - João Fábio Bertonha - Christopher Denis-Delacour - Maximiliano Fuentes Codera Anders Granås Kjøstvedt - John Paul Newman - Deborah Paci - Niccolò Pianciola - Spyridon Ploumidis - Wilko Graf Von Hardenberg

Comitato di redazione: Jacopo Bassi - Luca Bufarale - Gianluca Canè - Luca G. Manenti - Fausto Pietrancosta - Alessandro Salvador - Matteo Tomasoni - Luca Zuccolo 


\title{
17/ RECENSIONE: Gabriele DONATO, La violenza, la rivolta. Cronologia della lotta armata in Italia 1966-1988, Trieste, IRSML, 2018, 417 pp.
}

\author{
A cura di Alessandro CHEBAT
}

Il 2018 raccoglie in sé due anniversari importanti, il 1968 e il 1978, date che rappresentano episodi opposti ma legati tra loro da un sottile filo rosso: l'inizio della contestazione e l'uccisione di Aldo Moro'.

Troppo spesso il Sessantotto viene impropriamente legato all'emergere della lotta armata in Italia. Va sottolineato come la contestazione portasse con sé tematiche e istanze ben distanti dall'impianto ideologico e teorico di Brigate Rosse, Prima Linea e di tutta quella miriade di sigle che composero la galassia del terrorismo rosso negli anni Settanta e Ottanta.

Quella della lotta armata fu principalmente una storia operaia e comunista, come sottolineò a sua tempo Rossana Rossanda, secondo la quale, in riferimento ai brigatisti:

Vecchio o giovane che sia il tizio che maneggia la famosa Ibm, il suo schema è veterocomunismo puro. Cui innesta una conclusione che invece veterocomunista non è, la guerriglia ${ }^{2}$.

In sintesi, un armamentario ideologico legato ad un comunismo più classico ed ortodosso, ben distante dalla verve sessantottina, una ribellione di tipo generazionale che si proiettò verso il futuro senza avvertire la necessità di attingere al patrimonio conoscitivo di tutto ciò che era legato al "passato". Anche per quanto riguarda la pregiudiziale antifascista, i giovani contestatori italiani si affacciarono sul miracolo economico «contestando la metodologia della ricostruzione e

${ }^{1}$ SATTA, Vladimiro, I nemici della Repubblica. Storia degli anni di piombo, Milano, Rizzoli, 2016.

${ }^{2}$ ROSSANDA, Rossanda, «Il discorso sulla Dc», in il Manifesto, 28 marzo 1978, p. 1. 
della rappresentanza politica: contro i padri, perché hanno accettato o subito, o non sono riusciti a sconfiggere il fascismo» ${ }^{3}$.

Ciò cozzava contro la vulgata tradizionale del marxismo italiano del "popolo alla macchia" e del valore della lotta partigiana - imposta dal PCI per decenni - prediligendo delle più ampie (ma generiche) istanze anti-sistema, anti-capitaliste e contro la cultura e i valori tradizionali ${ }^{4}$.

$\mathrm{Fu}$ con l'emergere dell'antagonismo operaio nell'autunno caldo del Sessantanove, sull'onda lunga della contestazione giovanile, che avvenne la saldatura tra studenti e operai, per molti aspetti già annunciata nel 1967 con le tesi della Sapienza. L'incontro dei due movimenti, inizialmente molto differenti come origini e istanze, fece rifluire il movimento del Sessantotto su posizioni marxiste eterodosse - come il marxismo-leninismo e l'operaismo - ma che tuttavia affondavano le proprie origini nel socialismo classico con un richiamo rigoroso a Marx e Lenin. A ciò si aggiungevano suggestioni che provenivano da lontano, geograficamente e cronologicamente, come la guerriglia in America latina ${ }^{5}$ ma anche il mito della lotta partigiana come una Resistenza "tradita", la cui carica palingenetica era stata smorzata in nome della normalizzazione democratica del secondo dopoguerra. A questo scopo, è significativo che in questo stesso periodo figure "eretiche" della Resistenza e del comunismo italiano come Giovanni Pesce e Pietro Secchia, oltre a cercare un dialogo con i militanti della nuova sinistra, editarono diversi testi che ebbero notevole diffusione negli ambienti della sinistra radicale ${ }^{6}$.

In Italia - unico caso in Europa dove avvenne la fusione della protesta studentesca con quella operaia - si avvertì presto la necessità di attuare un salto di qualità rispetto alle proteste studentesche. Indicativo di questo stato d'animo risulta un articolo apparso su "Quaderni Piacentini» titolato Contro la falsa coscienza del movimento studentesco. Gli autori affermavano che la nascita del movimento studentesco aveva senza dubbio sollevato importanti problemi e annose questioni, senza però portare nessun profondo cambiamento né mutare i rapporti di forza tra le classi sociali ${ }^{7}$. Secondo i due autori la fascinazione eccessiva che le lotte in Sudamerica e in Asia orientale suscitavano negli studenti avrebbe provocato un grave equivoco cioè quello di «credere di fare già la rivoluzione perché facevano la contestazione»". La situazione di caos e di crisi istituzionale sarebbe perciò stata scambiata per un'azione prerivoluzionaria, mentre in realtà questi erano solo gli effetti superficiali generati dalle proteste studentesche e operaie.

\footnotetext{
${ }^{3}$ PARLATO, Giuseppe, Il sessantotto come data discrimine della storia d'Italia, in DE PASQUALE, Matilde, DOTOLI, Giovanni, SELVAGGIO, Mario (a cura di), I linguaggi del sessantotto, Roma, Apes, 2008, pp. 86-99.

${ }^{4}$ GIACHETTI, Diego, Un sessantotto e tre conflitti, Pisa, BFS, 2008, p. 67.

${ }^{5}$ MARIGHELLA, Carlos, Piccolo manuale della guerriglia urbana, Milano, Jaca Book, 1969.

${ }^{6}$ SECCHIA, Pietro, La guerriglia in Italia, Milano, Feltrinelli, 1969; ID., La Resistenza accusa 1945-1973, Milano, Mazzotta editore, 1973; ID., Lotta antifascista e giovani generazioni, Milano, La Pietra, 1973.

${ }^{7}$ CIAFALONI, Francesco, DONOLO, Carlo, «Contro la falsa coscienza del movimento studentesco», in Quaderni Piacentini, 38, 1969, pp. 30-41, p. 36.

${ }^{8}$ Ibidem.
} 
Questo aspetto fu fatto proprio dalle frange più radicali della nuova sinistra - tra le quali vi erano i membri del CPM, embrione delle Brigate Rosse - secondo cui lo scontro violento era una necessità sistematica e continua della lotta di classe ${ }^{9}$. Parafrasando Renato Curcio, siccome la prospettiva era quella della presa del potere, la lotta non poteva che evolvere in una guerriglia urbana nelle grandi città e negli stabilimenti industriali, distaccandosi dalle grandi proteste per compiere un salto qualitativo della lotta e, infine, presentarsi come unica e reale alternativa rispetto al PCI e alla sinistra extraparlamentare, i quali mancavano di concretezza rivoluzionaria.

Va altresì sottolineato come non fu solo il continuo stato di agitazione della "piazza" a determinare l'insorgere del terrorismo in Italia, ma anche due aspetti della politica governativa: da una parte il clamoroso fallimento del centrosinistra e il rapido spegnersi della sua spinta riformista nonostante le grandi attese; dall'altra la continua crescita del PCI, che tuttavia rimaneva un partito politicamente isolato ed escluso dalle stanze del potere. Paradossalmente, l'apertura della DC ad un compromesso con il PCI e il tentativo di Berlinguer di far uscire il partito dall'impasse con la graduale svolta moderata eurocomunista nell'ottica dei lottarmatisti rappresentavano un'evenienza mortale. Ciò avrebbe sancito l'abbandono di ogni "doppiezza" da parte del PCI e condotto ad un allontanamento della classe operaia da una (improbabile) prospettiva rivoluzionaria, vanificando la lotta clandestina di lunga durata ${ }^{10}$.

$\mathrm{Fu}$ questa la fase più dura e violenta, nel corso della quale la strategia delle Brigate Rosse fu caratterizzata dal cosiddetto "assalto al cuore dello stato", colpendo principalmente politici e tecnici. Va infatti sottolineato come la militanza armata all'interno delle fabbriche e la ricerca di un sostegno operaio alle proprie azioni (che in parte vi fu, ma sempre indiretto, mai fattuale) furono una ragion d'essere della lotta armata di sinistra. Una lotta che nella realtà dei fatti non alimentava altro che sé stessa, ma che comunque riusciva a trovare delle sue giustificazioni nel clima della strategia della tensione, innescata con la strage di piazza Fontana e la successiva morte dell'anarchico Pinelli. Da quel 12 dicembre 1969 si aprì un ventennio di stragi, attentati e uccisioni, dove alla lotta armata di sinistra si opposero lo stragismo dell'estrema destra, una gestione dell'ordine pubblico molto dura e non sempre avulsa da eccessi e il coinvolgimento di alti settori dello stato in episodi delittuosi e controversi, che tuttora attendono chiarezza ${ }^{11}$. Ancor più - all'indomani di Piazza Fontana - l'uscita del pamphlet La strage di stato ${ }^{12}$, col suo linguaggio mutuato dal gergo militare, spinse il conflitto verso la personalizzazione del nemico - fino a quel momento nelle sembianze di anonimi poliziotti o fascisti - e ora individuato nel commissario

\footnotetext{
${ }^{9}$ CAVALLINI, Massimo, Il terrorismo in fabbrica: interviste, Roma, Editori Riuniti, 1978, p. 125.

${ }^{10}$ SECHI, Salvatore, Compagno cittadino: il PCI tra via parlamentare e lotta armata, Soveria Mannelli, Rubbettino, 2006, pp. 53-54.

${ }^{11}$ BALESTRINI, Nanni, MORONI, Primo, L'orda d'oro: 1968-1977, Milano, Feltrinelli, 1997; DE LUNA, Giovanni, Le ragioni di un decennio: 1969-1979, Milano, Feltrinelli, 2011.

${ }^{12}$ DI GIOVANNI, Eduardo, LIGINI, Marco, La strage di Stato: controinchiesta, Roma, Samonà e Savelli, 1970.
} 
Calabresi, prima vittima "istituzionale" di quella escalation di violenza. Va sottolineato come la strategia della tensione portò con sé un altro elemento che segnò il passaggio dalle "armi della retorica alla retorica delle armi": la "psicosi del colpo di stato" ovvero la percezione diffusa che ci fosse il rischio che l'avanzata della sinistra e l'antagonismo operaio e studentesco potessero innescare una svolta autoritaria in Italia per mano delle frange più reazionarie del paese. Di queste istanze si fecero portavoce non solo gli intellettuali engagés o i movimenti extraparlamentari, ma anche ex partigiani come Gianbattista Lazagna e il professor Guido Quazza $^{13}$, oppure personaggi noti come Giangiacomo Feltrinelli, che tra il 1968 e il 1969, diede alle stampe diversi pamphlet nei quali denunciava la concreta minaccia di una deriva autoritaria in Italia ${ }^{14}$.

Sarebbe però fuorviante limitare il fenomeno del terrorismo in Italia unicamente a ragioni di politica interna; esso infatti tese a legarsi e a trovare ragion d'essere anche in relazione ad eventi politici internazionali in chiave anti-capitalista e anti-americana. Gli esempi sono innumerevoli, tuttavia è sufficiente citare la guerra del Vietnam, la dittatura dei colonnelli in Grecia, la guerriglia in Sud America, il golpe cileno del 1973 e la causa palestinese, per comprendere il peso che ebbe il quadro internazionale nel determinare gli eventi. Il terzomondismo, l'internazionalizzazione della lotta armata, furono un fenomeno proprio del periodo e riguardò non solo l'Italia ma anche la Germania, con la RAF. Una commistione di suggestioni passate e presenti che De Bernardi definisce un corto circuito ideologico che metteva insieme e confondeva il Che e i partigiani, Arafat e Lazzagna, la guerriglia vietnamita e le brigate Garibaldi, l'incendio del Reichstag con l'attentato all'Italicus, e che condussero alcune frange più radicali del movimento ad un salto di qualità verso la lotta armata clandestina ${ }^{15}$.

Nel complesso un clima da "guerra civile a bassa intensità" ${ }^{\text {, }}$, nel quale l'uso della violenza non fu monopolizzato dalle azioni dei gruppi armati clandestini, bensì quotidiano e diffuso in ogni ambito $^{17}$ : perpetrata in modo arbitrario dai gruppi extraparlamentari o da singoli militanti, soprattutto con il movimento dell'Autonomia nel 1977. Tuttavia, la violenza non si concretizzava solamente in scontri, uccisioni o pestaggi - come nei casi del rogo di Primavalle o l'uccisione dei militanti del MSI Sergio Ramelli e Carlo Falvella - ma anche in pubbliche umiliazioni degli

\footnotetext{
${ }^{13}$ Quazza fu impegnato in un'originale opera di analisi della Resistenza, declinandola come una sorta di antifascismo esistenziale e prepolitico molto simile a quello della sinistra extraparlamentare. Cfr. QUAZZA, Guido, Resistenza e storia d'Italia: problemi e ipotesi di ricerca, Milano, Feltrinelli, 1977.

${ }^{14}$ FELTRINELLI, Giangiacomo, Persiste la minaccia di un colpo di stato in Italia!, Milano, Feltrinelli, 1968; ID., Estate 1969, Milano, Feltrinelli, 1969.

${ }^{15}$ DE BERNARDI, Alberto, RAPINI, Andrea, Discorso sull'antifascismo, Milano, Bruno Mondadori, 2007, p. 198.

${ }^{16}$ ZAVOLI, Sergio, La notte della Repubblica, Milano, Mondadori, 2009.

${ }^{17}$ RAPINI, Andrea, Antifascismo e cittadinanza, Bologna, Bononia University Press, 2005.
} 
avversari politici: a titolo d'esempio la gogna proletaria di Trento ai danni dei missini Mitolo e Del Piccolo, il 30 luglio del 1970, che inaugurò una serie di eventi similari in tutta Italia ${ }^{18}$.

Una violenza che non fu solo di fatto, ma anche diffusa a livello iconografico: si pensi alle copertine della rivista «Rosso», dove provocatoriamente venivano mostrate immagini di scontri tra militanti e poliziotti. L'aggettivazione violenta fu presente anche negli slogan - come «Vietcong vince perché spara» del post-68, «Democrazia è il fucile in spalla agli operai» di Potere Operaio, oppure la «Compagna P38», accompagnato dal gesto con la mano, diffuso tra il movimento dell'Autonomia - nonché nella musica ${ }^{19}$.

E proprio dal ritornello di una famosa canzone del periodo di Alfredo Bandelli che prende il titolo questa cronologia degli Anni di piombo a cura di Gabriele Donato, La violenza, la rivolta. Il testo, come chiarisce l'autore nell'introduzione, trae diretta origine dal precedente volume $L a$ lotta è armata e da una problematica ben presente in ogni testo che prenda in considerazione il ventennio 1966-1988 ovvero non solo dare ordine ai fatti in se, bensì inquadrarli nel contesto più ampio della storia sociale, politica, culturale e delle relazioni internazionali, dai quali gli eventi stessi prendevano le proprie mosse e ragion d'essere ${ }^{20}$. Il testo di Donato risponde a questa problematica, fornendo allo studioso una completa sequenza di eventi dall'omicidio del militante socialista Paolo Rossi - il 27 aprile 1966 - fino all'attentato a Roberto Ruffilli il 17 aprile del 1988, che chiuse la stagione della Brigate Rosse storiche. Una proposta di datazione non casuale e che si basa sia su fatti oggettivi, ovvero una spirale di violenza ben delimitata dai due episodi in questione, sia sul contesto sociale, politico e culturale di questo ventennio ${ }^{21}$. Ne esce

una linea del tempo strutturata su diversi piani a rappresentare le cinque macro-dimensioni entro le quali sono stati raggruppati i fatti considerati: lotta armata e terrorismo di sinistra; strategia della tensione e terrorismo di destra; organizzazioni, iniziative e dibattiti dell'estrema sinistra; quadro internazionale [...]; quadro nazionale $[. . .]^{22}$.

Infatti, tra queste due date l'autore non cita solo gli eventi italiani o internazionali più rilevanti, gli attentati o gli episodi di violenza, ma anche la pubblicazione di testi e pamphlet, le risoluzioni dei congressi dei partiti o dei movimenti politici, gli interventi e le azioni dei protagonisti di allora in relazione ai fatti di cronaca, tutti elementi che hanno contribuito a tracciare l'epoca presa in considerazione. A questo scopo merita una menzione particolare la

\footnotetext{
${ }^{18}$ «Ieri oggi sempre Resistenza: MSI fuorilegge», supplemento a Lotta Continua, 27 giugno 1974, p. 2.

${ }^{19}$ ZAVARONI, Pierluigi, Caduti e memoria nella lotta politica, Milano, Franco Angeli, 2010, pp. 50-51.

${ }^{20}$ DONATO, Gabriele, La lotta è armata, Roma, DeriveApprodi, 2014 [ed. orig.: Trieste, Irsml, 2012].

${ }^{21}$ Alla base della proposta di datazione l'autore riporta le riflessioni proposta da GALFRÉ, Monica, La guerra è finita, Roma-Bari, Laterza, 2014.

${ }_{22}^{22}$ DONATO, Gabriele, La violenza, la rivolta. Cronologia della lotta armata in Italia 1966-1988, Trieste, Irsml, 2018, p. 8.
} 
veste grafica, non solo da un punto di vista puramente estetico bensì per il costante richiamo alla pubblicistica di allora con l'inserimento di stralci di Potere Operaio, Lotta Continua, ecc.

Da sottolineare come - malgrado il termine "lotta armata" faccia riferimento alla galassia dell'estrema sinistra - l'elenco degli eventi prenda ampiamente in considerazione anche figure, atti ed eventi afferenti al terrorismo di estrema destra. Ciò, sia in relazione agli innumerevoli episodi di violenza politica comune perpetrata dai militanti neofascisti ai danni di quelli di sinistra, sia riguardo alla strategia stragista che ha legato l'estrema destra ai settori "deviati" dello stato, sia nel tentativo di emulazione della lotta armata di sinistra da parte dei NAR.

Risulta inoltre di grande utilità il cospicuo indice dei nomi e dei luoghi, elemento spesso trascurato nelle cronologie, ma che in realtà è fondamentale per inquadrare e spiegare un determinato evento permettendo un ampliamento delle prospettive interpretative. Questo, soprattutto in un quadro come quello degli anni di piombo, che è spesso necessario affrontare con gli strumenti offerti dalla storia sociale e culturale, accostando nomi e luoghi ai fatti.

Per quanto riguarda la questione delle fonti, l'autore si è rivolto in primis a materiale documentario costituito dai quotidiani dell'epoca conservati nel fondo «Renzo Pincherle», presso l'archivio dell'IRSML di Trieste. Tuttavia, l'autore ha ampiamente usufruito anche alle numerose cronologie disponibili online, in particolare quello della Fondazione Luigi Cipriani. Sempre online l'autore ha potuto consultare quotidiani, documenti, pamphlet, volantini e manifesti afferenti ai vari gruppi della nuova sinistra, liberamente visionabili su molti siti. Ciò a dimostrazione di come archivi online e offline possano essere elementi complementari e non opposti che, sapientemente impiegati come in questo caso, sono andati ad ovviare alla mancanza di una cronologia della lotta armata nella produzione editoriale italiana.

Unico aspetto che avrebbe meritato un approfondimento è il periodo tra il 1960 e il 1965, la cosiddetta fase dei "giovani prima della rivolta" ${ }^{23}$. In questo quinquennio avvennero una serie di eventi decisivi utili per spiegare le ragioni profonde sia di coloro che decisero di imbracciare le armi sia di quanti percorsero la via dell'antifascismo militante. I disordini del luglio 1960, contro il congresso del MSI a Genova e il governo Tambroni ne sono un esempio. In particolare i morti durante gli scontri a Reggio Emilia divennero martiri di una "nuova" Resistenza contro il "nuovo" fascismo istituzionalizzato di Tambroni. L'episodio fu reso celebre dalla canzone Per i morti di Reggio Emilia di Fausto Amodei, incisa nello stesso anno per «Cantacronache» e poi ripresa nel 1974 dagli Stormy Six, gruppo legato agli ambienti della sinistra radicale. Di fatto, da quel momento in poi l'anticomunismo smise di essere il valore fondamentale per la Repubblica e divenne minoritario rispetto all'antifascismo ${ }^{24}$.

\footnotetext{
${ }^{23}$ GHIONE, Paola, GRISPIGNI, Marco, Giovani prima della rivolta, Roma, Manifestolibri, 1998.

${ }^{24}$ GINSBORG, Paul, Storia d'Italia dal dopoguerra ad oggi, Torino, Einaudi, 2006, p. 348.
} 
Strettamente legata a tale episodio fu la contestazione della Resistenza "tricolore" durante la celebrazione del suo ventennale, il 9 maggio 1965. In quell'occasione agli ex partigiani fu ordinato di indossare una fascia tricolore al posto dei fazzoletti rossi delle brigate Garibaldi. I circa 80mila presenti, per la prima volta ruppero la sacralità delle celebrazioni ufficiali, contestando la solennità dei discorsi istituzionali e organizzando un corteo di protesta contro la guerra del Vietnam. Questo processo di reazione e rifiuto di una Resistenza che molti definirono "imbalsamata", "depotenziata", "innocua" ed elevata a mito fondativo nazionale - cioè non più in grado di creare fratture e portare avanti nuove battaglie politiche e sociali - rappresentò una delle chiavi di lettura attraverso le quali si mosse una fetta importante dell'antifascismo militante e una parte del primo lottarmatismo italiano.

Nello stesso anno di Franco Fortini pubblicava un saggio che raccoglieva in sé molte delle tematiche che verranno fatte proprie dal movimento del Sessantotto prima e dall'estrema sinistra poi $^{25}$. In esso Fortini ipotizzava come la convergenza tra intellettuali rivoluzionari e intellettuali democratico-borghesi su una piattaforma umanistica e progressista avesse diluito la lotta di classe nella difesa generica dei diritti dell'uomo e della civiltà contro la barbarie del fascismo. L'intervento di tali intellettuali fece perciò coincidere l'antifascismo con un comunismo non più votato al "ribaltamento rivoluzionario" della società, ma ad un'astratta difesa dei diritti acquisiti o da acquisire all'interno di un sistema capitalista ${ }^{26}$. Secondo Fortini l'unico e vero antifascismo doveva essere quello di classe e anticapitalistico, volto a costruire una società nuova ove non vi fosse posto per qualsiasi "residuato" di fascismo.

Tuttavia, il periodo 1960-65 fu decisivo non solo nella rilettura critica del passato, ma anche nell'analisi delle dinamiche interne di un elemento centrale nell'innescare il ventennio degli anni di piombo: la classe operaia. Nel 1961 uscì Lotte operaie nello sviluppo capitalistico, il primo numero della rivista "Quaderni Rossi», fondata da Tronti, Negri e Panzieri, che sarà la culla del pensiero operaista. Negli anni successivi Lotta Continua e Potere Operaio si ispirarono a tali esperienze, mentre più dibattuta fu l'influenza dell'operaismo sui movimenti armati ${ }^{27}$.

In realtà l'operaio massa di Tronti, ovvero l'operaio dequalificato della catena di montaggio, privo di competenze e responsabile di una piccola funzione all'interno del processo automatizzato, manifestò la sua prima fiammata di antagonismo ben prima del '69. Il 7 luglio del 1962 a Torino, in piazza Statuto, si consumò una rivolta che anticipò l'Autunno caldo. Aderendo ad uno sciopero per il rinnovo dei contratti, gli operai massa meridionali, con uno slancio

\footnotetext{
${ }^{25}$ FORTINI, Franco, Mandato degli scrittori e fine dell'antifascismo, in ID., Verifica dei poteri, Milano, Il Saggiatore, 1965.

${ }^{26}$ RAPINI, Andrea, Antifascismo e Resistenza nelle riviste della nuova sinistra, in ADAGIO, Carmelo, CERRATO, Rocco, URSO, Simona (a cura di), Il lungo decennio. L'Italia prima del '68, Verona, Cierre, 1999, pp. 373-411, pp. 376-378.

${ }^{27}$ Cfr. BORIO, Guido, POZZI Francesca, ROGGERO, Gigi, Gli operaisti: autobiografie dei cattivi maestri, Roma, DeriveApprodi, 2005.
} 
prepolitico, diedero vita a disordini e scontri con la polizia, scavalcando il sindacato e il Partito comunista che furono oggetto di aspre critiche. Un PCI che, colto di sorpresa da una radicalità che non era in grado di controllare, definirà gli scontri «tentativi teppistici e provocatori», ed i manifestanti «elementi incontrollati ed esasperati», anticipando quel distacco tra partito e nuova sinistra che caratterizzò gli anni Settanta ${ }^{28}$.

Infine, merita di essere citato un ultimo episodio, tanto significativo quanto sconosciuto, ovvero l'incruento rapimento a Milano del console spagnolo Isu Elias, il 28 settembre 1962, da parte di un gruppo di anarchici, come segno di protesta contro la possibile condanna a morte di tre dissidenti catalani accusati di terrorismo. Fu il primo sequestro politico in Italia, motivato da una solidarietà con le lotte internazionali che sarà propria della lotta armata negli anni Settanta. Una solidarietà che nell'ottobre 1962, a Milano, costò la vita allo studente Giovanni Ardizzone, morto negli scontri scoppiati durante una manifestazione per la crisi dei missili di Cuba ${ }^{29}$.

Per concludere, se è vero che la scansione cronologica degli eventi storici non permette di spiegarli e interpretarli, è altrettanto vero che la violenza politica negli anni Settanta e Ottanta va studiata attraverso molteplici griglie interpretative, per la cui lettura è necessaria la presenza di una cronologia di eventi che raccolga tutti i vari fattori interni alla società italiana, nonché quelli internazionali o politico-culturali che hanno segnato un'epoca. Il testo di Gabriele Donato si presenta come una risposta utile a questa problematica, inquadrando il periodo della lotta armata non attraverso una analisi storiografica o sociologica, ma ponendo al centro ciò che sta a monte dello studio, ovvero gli eventi che hanno contestualizzato tale violenza.

\footnotetext{
${ }^{28}$ LANZARDO, Dario, La rivolta di Piazza Statuto, Milano, Feltrinelli, 1979.

${ }^{29}$ BERMANI, Cesare, Il nemico interno. Guerra civile e lotta di classe in Italia (1943-1976), Roma, Odradek, $1997, \mathrm{pp}$. 21-24.
} 


\section{L'AUTORE}

Alessandro CHEBAT ha studiato presso l'università degli studi di Milano, ottenendo la laurea triennale in Scienze Storiche e ha poi conseguito la laurea magistrale in scienze storiche e forme della memoria all'università di Trento. Ha preso parte al progetto "La Grande Guerra + 100", ha collaborato a varie iniziative editoriali con Textus e Salerno, nonché all'organizzazione della mostra "La guerra che verrà non è la prima" (Mart-Rovereto). Attualmente è educatore presso un CFP in provincia di Trento.

URL: < http://www.studistorici.com/progett/autori/\#Chebat > 